\title{
Efectos de la crisis económica en el gasto en transporte de las familias españolas: diferencias según regiones y niveles de ingreso
}

\author{
Rocío Cascajo Jiménez \\ Investigadora, TRANSyT-UPM, España \\ Aldara Tadeo Martín \\ Consultora de transportes, INECO, España \\ Andrés Monzón de Cáceres \\ Catedrático de Transportes, UPM, España
}

\begin{abstract}
RESUMEN
La crisis económica y financiera que comenzó en el año 2007 y en la que aún estamos inmersos ha provocado numerosos cambios socioeconómicos en España. El aumento de la tasa de paro y la reducción de la renta disponible por hogar están provocando que los hogares españoles reduzcan sus gastos medios anuales.
\end{abstract}

El objetivo de la ponencia es investigar cómo la crisis económica y financiera ha afectado a los hogares españoles y de qué manera esto está afectando a la forma en la que los españoles se desplazan. Para ello se analizarán los gastos de los hogares en los años 2007 (justo al inicio de la crisis) y 2012, a nivel nacional y en 6 Comunidades Autónomas. Se considerarán los gastos en general, centrándonos en los gastos dedicados al transporte. Los datos provienen de la encuesta de presupuestos familiares que se realiza anualmente a una muestra de hogares españoles. La investigación se enmarca en el proyecto CIME, La Crise et ses Impacts sur la Mobilité en Espagne. Mesures, analyses et enseignements pour le cas français, financiado por el gobierno francés y desarrollado por un equipo franco-español.

Los principales resultados que surgen son que, en general, los hogares españoles reducen sus gastos en transporte personal (compra y uso de coche) entre 2007 y 2012, mientras que en el caso del gasto en servicios de transporte público, los resultados son más dependientes del nivel de renta de los hogares y de la región analizada.

\section{INTRODUCCIÓN}

La crisis financiera que se inició en el verano de 2007 representa un punto de inflexión en la evolución de diversas variables económicas. Sin embargo, no todos los países se han visto afectados por igual: mientras que las economías de los países desarrollados se vieron dañadas sustancialmente, los países emergentes hicieron frente relativamente bien. En la zona UE15, la mayoría de los países experimentaron una ligera desaceleración en la tasa de crecimiento en 2008, seguido de una disminución importante en el año 2009. En general, esta caída fue más pronunciada en los países con altos valores anteriores a la crisis, como el Reino Unido $(-18,4 \%)$, Suecia $(-17,2 \%)$, Irlanda $(-15,3 \%)$ y Dinamarca $(-10,2 \%)$. Por el contrario, Portugal y Grecia registraron las caídas más suaves, con -7,2\% y -6,4\%, respectivamente, entre 2008 y 2009 (EC, 2009). Aunque parecía que el crecimiento se recuperaba en 2011, el PIB per cápita cayó de nuevo en 2012, evitando así que la UE-15 recuperase el nivel de 2008. Fuera de Europa, otros países desarrollados como Japón y Estados Unidos no se han visto tan afectados por la crisis. Países emergentes como Brasil, Rusia, India, China y México, a pesar de estar afectados por la crisis, ahora están creciendo por encima de los niveles del PIB per cápita de 2008 (Banco Mundial, 2014). Parece, por 
tanto, que Europa es la región que más ha sido afectada por la crisis actual. Las cifras de desempleo muestran que en los países del norte de la UE 15 las tasas se mantuvieron por debajo del $9 \%$ en 2013. La situación es muy diferente en los países del sur de Europa, donde las tasas de desempleo son el doble que en 2008, y en particular en Grecia y España, cuyas cifras de desempleo fueron de más del $26 \%$ en 2013. La renta bruta disponible per cápita revela que, si bien la mayor parte de los países de la UE 15 del norte y central han aumentado sus ingresos por encima de los niveles previos a la crisis, los países del sur todavía están luchando por hacerlo (Eurostat, 2014).

Se han realizado muchas investigaciones sobre la influencia de diferentes factores como la renta disponible, las tarifas o el nivel de motorización sobre la demanda de transporte de pasajeros (Nijkamp and Peppong, 1998; Dargay, 2001; Bresson et al, 2003; Paulley et al, 2006; Dargay, 2007; Holmgren, 2007; Taylor et al, 2009; Frondel and Vance, 2011); sin embargo, poco se ha encontrado sobre los efectos de la actual crisis en el gasto en transporte (Martínez et al, 2014). Por este motivo, y en vista de las consecuencias graves de esta crisis en España, se llevó a cabo una investigación enmarcada en el proyecto CIME, La Crise et ses Impacts sur la Mobilité en Espagne. Mesures, analyses et enseignements pour le cas français, financiado por el gobierno francés y desarrollado por un equipo franco-español.

El objetivo de este trabajo es analizar cómo estos cambios profundos y acelerados en la economía de España han afectado a gastos de los hogares en general, y en el transporte en particular, y cómo la crisis económica ha afectado de manera diferente la forma en que los ciudadanos gastan sus ingresos en base a sus niveles de renta. El análisis se basa en la Encuesta de Presupuestos Familiares (EPF), llevada a cabo anualmente por el Instituto Nacional de Estadística (INE). El estudio se centra en seis regiones españolas y en el conjunto de España.

\section{METODOLOGÍA}

\subsection{Datos utilizados}

Los datos utilizados para realizar el estudio se basan en los gastos de transporte de los hogares españoles entre 2007 y 2012, extraídos de la Encuesta de Presupuestos Familiares (EPF). La EPF se publica anualmente por el INE y proporciona datos sobre el gasto en consumo de los hogares españoles, y revela la forma en que se distribuye entre las diferentes categorías de consumo.

La clasificación utilizada para ordenar los gastos es la COICOP ${ }^{1}$. Está estructurado en doce grupos grandes, siendo el Grupo 7 el que contiene los gastos de transporte, revelando el presupuesto que dedican los hogares al transporte cada año. Este estudio se centra en el presupuesto dedicado al transporte de viajeros por carretera, por lo que se analizarán los siguientes gastos:

- Compra de automóviles (código 0711): adquisición de coches, furgonetas, vehículos todo terreno destinados al transporte de los miembros del hogar. Incluye la adquisición, registro e IVA de vehículos nuevos y de segunda mano.

- Utilización de vehículos personales (código 072): gasto asociado a la reparación, mantenimiento e inspección de los vehículos; combustible y aceite; pago de aparcamientos públicos y peajes; y la obtención y renovación de los permisos de conducir. Dentro de esta investigación nos referiremos a los gastos del uso del coche.

- Servicios de transporte (correspondiente al código 073): en este estudio se han

1 COICOP son las siglas de "classification of individual consumption by purpose". 
considerado los gastos incluidos en los códigos de cinco dígitos 07311, 07313, 07321, 07323 y 0735 , que se corresponden con las tarifas y billetes multimodales dedicados a los servicios de transporte público en áreas urbanas y metropolitanas, incluyendo metro, tranvía, tren de cercanías, autobuses urbanos, taxis y transporte escolar, y los gastos dedicados a los servicios de transporte interurbano (que contienen los códigos 07312 y 07322), que incluyen trenes y autobuses cubriendo distancias de más de 50 kilómetros.

Los pasos para analizar la EPF fueron los siguientes: 1) Clasificación de los hogares por región; 2) Clasificación de los hogares en cada región por el tamaño del municipio (la EPF establece los siguientes intervalos: <10.000 habitantes; 10.000 - 20.000 habitantes; 20.000 50.000 habitantes; 50.000 - 100.000 habitantes y $\geq 100.000$ habitantes); 3) Agrupación de los hogares de acuerdo a los ingresos netos al mes (los cuatro grupos de ingresos netos considerados en este estudio son: $<1.000 € ; 1.000-2.000 € ; 2.000-3.000 € ; \geq 3.000 €)$.

Para este estudio, se analizaron los datos de la EPF durante dos años: 2012 y 2007, el último año antes de que los efectos de la crisis se hicieran evidentes en España. El año base se estableció a la vista de la evolución de algunos indicadores macroeconómicos, tales como la tasa de desempleo o el PIB. Para la comparación de datos de los dos años, los gastos de 2007 se han actualizado a 2012 de acuerdo con la variación del IPC general desde diciembre de 2007 a diciembre 2012.

\subsection{Descripción de los casos de estudio}

La investigación se centra en los municipios españoles con 100.000 o más habitantes (63 ciudades), donde los servicios de transporte público urbano y metropolitano se vuelven importantes. Se han elegido 6 de las 17 Comunidades Autónomas para llevar a cabo el estudio: la Comunidad de Madrid, Cataluña, Comunidad Valenciana, Andalucía, País Vasco y Navarra. Las cinco primeras se eligieron por su importancia en la economía española, evaluada a través de su contribución al PIB español. De acuerdo con cifras de 2012, estas cinco regiones representaron el 66\% del PIB nacional. Además, estas regiones también son significativas debido a su gran población, tanto en términos absolutos como en relación a su área. Cinco de las seis ciudades más pobladas (Madrid, Barcelona, Valencia, Sevilla y Málaga) y 42 de las 63 ciudades con más de 100.000 habitantes en España pertenecen a estas regiones. La sexta región -Navarra- se incluye por diferentes razones; con tan sólo 600.000 habitantes es una de las regiones con el mayor PIB per cápita. A diferencia de las otras regiones seleccionadas, sólo hay un municipio con más de 100.000 habitantes en Navarra: la ciudad de Pamplona.

Si hay un indicador cuya evolución se ha visto afectada de manera significativa por la crisis actual es la tasa de desempleo. Así, en la Figura 1 se observa que 2007 fue el año con menor tasa de desempleo para todas las regiones. A excepción de Andalucía, cuya tasa de desempleo se ha mantenido históricamente por encima de la media nacional, las otras cinco regiones tuvieron tasas inferiores al $10 \%$ en el año 2007. Después de cinco años, todas las tasas de desempleo están por encima del 15\%, siendo las más altas las de Cataluña (22,5\%), Comunidad Valenciana (27,2\%) y Andalucía (34,3\%).

Además del desempleo, el PIB también ha disminuido a causa de la crisis. El PIB per cápita nacional alcanza su máximo en $2008(24.300 € / \mathrm{hab})$ y desde entonces empieza a disminuir hasta el año 2014, que parece que empieza a recuperarse. A nivel regional, algunas regiones empiezan a notar antes la crisis, como Andalucía, Madrid o Cataluña, que presentan su valor de PIB regional más alto en 2007. 


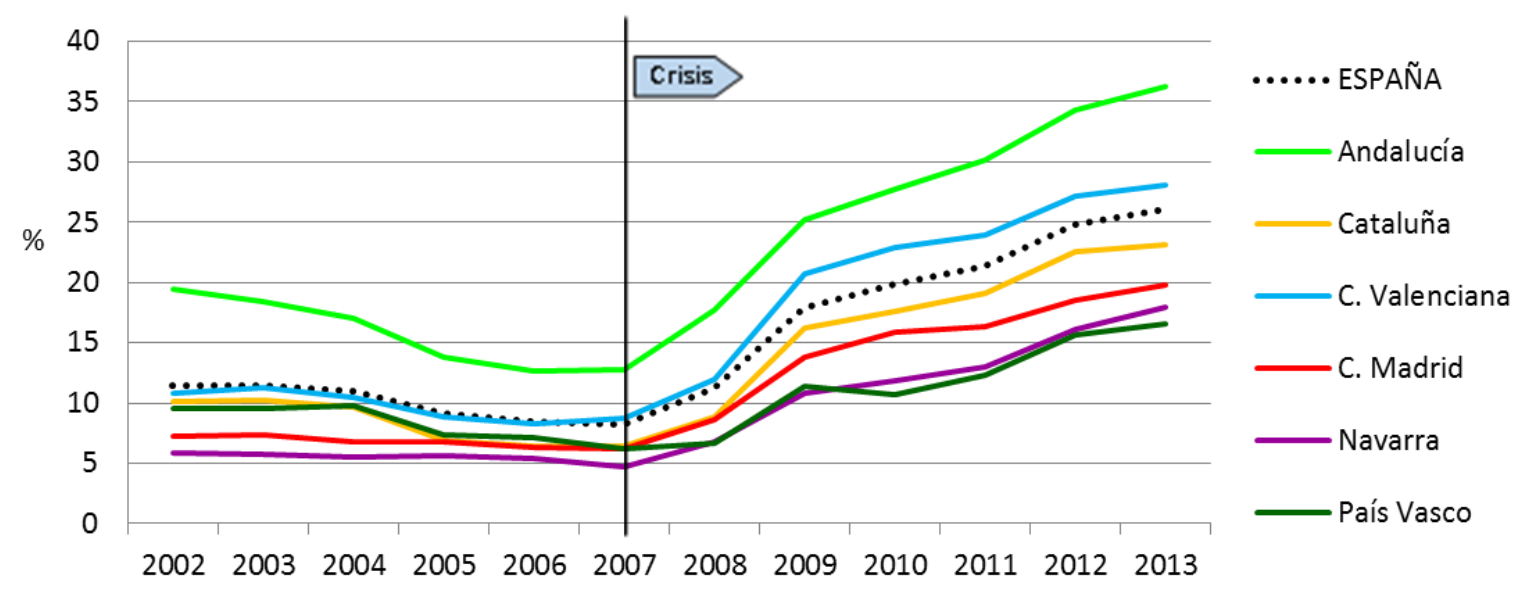

Fig. 1 - Tasa de desempleo en las regiones seleccionadas (2002-2013)

\section{RESULTADOS Y DISCUSIÓN}

Esta sección presenta los gastos de los hogares en transporte entre 2007 y 2012. En primer lugar, se compara el gasto de los hogares en transporte terrestre con el gasto total de los hogares. En segundo lugar, el análisis se centra en los gastos en transporte personal frente a los gastos en transporte público. Por último, los gastos en transporte se dividen en diferentes categorías y se analizan individualmente. En todas las secciones, los gastos por hogar de las seis regiones se comparan según niveles de ingresos.

\subsection{Proporción del gasto familiar dedicado al transporte}

El gasto medio anual por hogar en España fue de $32.000 €$ en 2007 y $28.152 €$ en 2012 (INE), por lo que la reducción de gastos entre 2007 y 2012, teniendo en cuenta los precios constantes, fue del $20,7 \%$. El gasto medio anual por hogar en transporte (transporte personal y servicios de transporte público) fue de $4.593 €$ en 2007 y $3.321 €$ en 2012, siendo la reducción de gastos en transporte, teniendo en cuenta los precios constantes, del 34,8\%. Estas cifras indican que la reducción de los gastos por hogar en transporte es mayor que la reducción en el gasto total en el período de recesión económica, y también mayor que otros grupos de gastos como alimentación y vivienda (Cascajo et al 2014).

Es interesante analizar la proporción del gasto total de los hogares dedicada al transporte según niveles de ingreso (Tabla 1). En primer lugar, los hogares con ingresos mensuales inferiores a $1.000 €$ incrementan su gasto total en transporte en 2012 con respecto a 2007. También es cierto para los gastos en transporte personal, pasando de 4,3\% en 2007 a 4,9\% en 2012, y en el gasto en servicios de transporte público, pasando de 0,9 a $1,5 \%$. Lo contrario ocurre en el resto de grupos de niveles de ingreso: el porcentaje de gasto de los hogares en transporte personal disminuye en 2012, mientras que la proporción del gasto de los hogares en servicios de transporte público aumenta en 2012.

En segundo lugar, se observa que el porcentaje de gasto en transporte personal de los hogares con ingresos mensuales superiores a $2.000 €$ está entre 9,5 y $12,5 \%$, mientras que en los hogares más pobres se sitúa entre 4-5\%. Sin embargo, cuando se trata del gasto en servicios de transporte público, el porcentaje es similar en todos los hogares, 0,9-1,5\%, independientemente de su nivel de ingresos. Una primera conclusión de este análisis es que la proporción del gasto dedicada al transporte personal depende del nivel de ingresos de los hogares, siendo mayor en los hogares con mayores ingresos. Sin embargo, no es cierto para los gastos en servicios de transporte público, ya que la proporción de gasto es independiente 
del nivel de ingresos del hogar.

\begin{tabular}{lrrrrrr}
\hline \multirow{2}{*}{$\begin{array}{l}\text { Nivel de } \\
\text { Ingresos }\end{array}$} & \multicolumn{2}{c}{$\begin{array}{c}\text { Gasto en transporte } \\
\text { personal }\end{array}$} & \multicolumn{2}{c}{$\begin{array}{c}\text { Gasto en servicios de } \\
\text { transporte público }\end{array}$} & \multicolumn{2}{c}{$\begin{array}{c}\text { Gasto Total en } \\
\text { transporte }\end{array}$} \\
\cline { 2 - 7 } & $\mathbf{2 0 0 7}$ & $\mathbf{2 0 1 2}$ & $\mathbf{2 0 0 7}$ & $\mathbf{2 0 1 2}$ & $\mathbf{2 0 0 7}$ & $\mathbf{2 0 1 2}$ \\
\hline$<\mathbf{1 . 0 0 0} €$ & $4,3 \%$ & $4,9 \%$ & $0,9 \%$ & $1,5 \%$ & $5,1 \%$ & $6,3 \%$ \\
\hline $\mathbf{1 . 0 0 0}-\mathbf{2 . 0 0 0} \boldsymbol{\epsilon}$ & $10,7 \%$ & $7,6 \%$ & $1,1 \%$ & $1,5 \%$ & $11,8 \%$ & $9,1 \%$ \\
\hline $\mathbf{2 . 0 0 0}-\mathbf{3 . 0 0 0} \boldsymbol{\epsilon}$ & $12,5 \%$ & $9,5 \%$ & $1,1 \%$ & $1,4 \%$ & $13,6 \%$ & $10,8 \%$ \\
\hline$>\mathbf{3 . 0 0 0} \boldsymbol{E}$ & $12,3 \%$ & $10,2 \%$ & $1,2 \%$ & $1,3 \%$ & $13,5 \%$ & $11,5 \%$ \\
\hline
\end{tabular}

Tabla 1. Proporción del gasto total dedicado al transporte (\%) de los hogares españoles en 2007 y 2012

\subsection{Evolución de los gastos en transporte personal y en servicios de transporte público}

En la Figura 2 se muestra el gasto medio anual dedicado al transporte personal (coche) y el dedicado a los servicios de transporte público (TP) en 2007 y 2012, por región y a nivel nacional. Se observa que el gasto dedicado a los servicios de transporte público en los dos años, 2007 y 2012, es inferior al realizado en transporte personal. También se aprecia que hay una disminución en el gasto en transporte personal entre 2007 y 2012 en todas las regiones. Sin embargo, el gasto en servicios de transporte público aumenta en casi todas las regiones, a excepción del País Vasco, que muestra una reducción de alrededor del $8 \%$ en 2012.

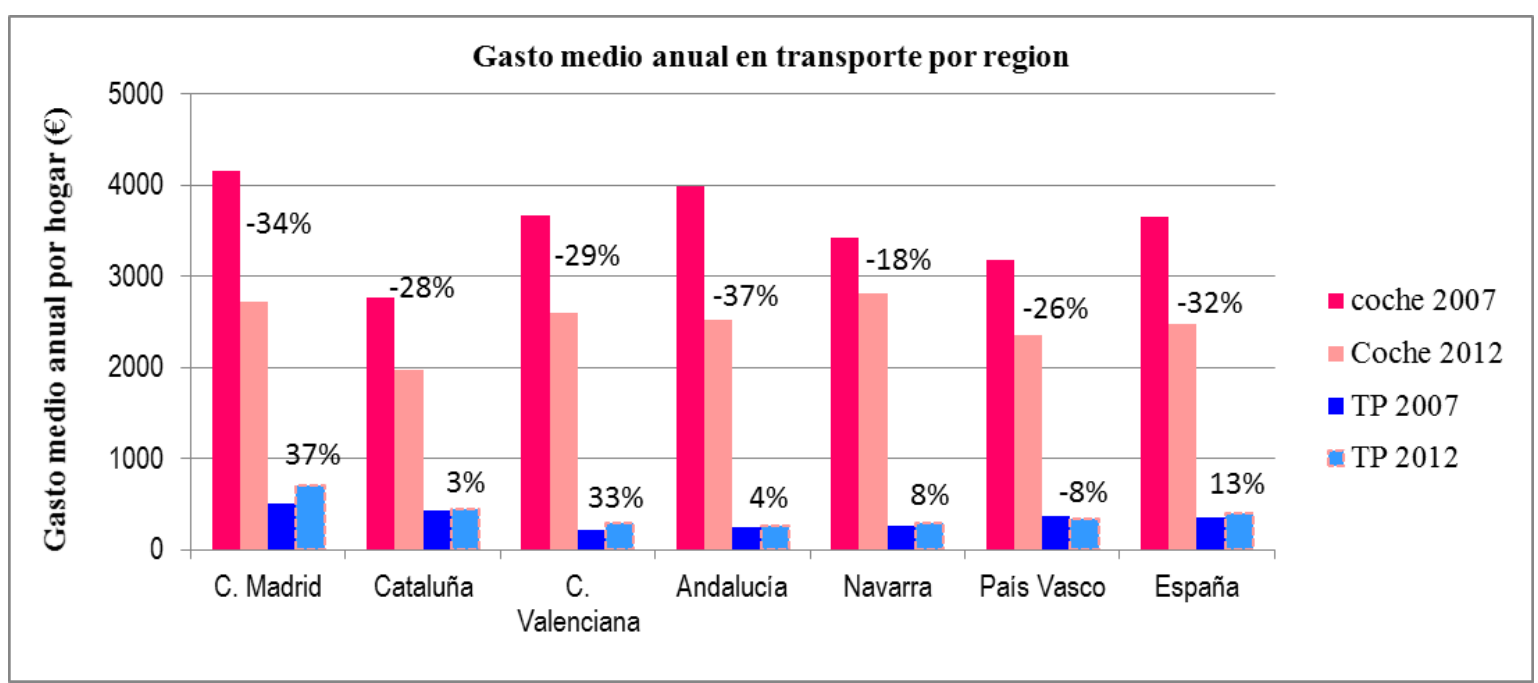

Fig. 2 - Los gastos de transporte personal contra el transporte público por hogar en las regiones seleccionadas en 2007 y 2012

La Figura 3 presenta el gasto familiar anual en transporte personal y en servicios de transporte público a nivel nacional por nivel de ingresos. Se observa que a mayor nivel de ingresos, mayor es el gasto que dedican al transporte. Además, se aprecia una disminución general de los gastos en transporte personal entre 2007 y 2012, que varía según el nivel de ingresos: así, los hogares con ingresos netos mensuales por debajo de $1.000 €$, presentan un mayor gasto en 2012 que en 2007. Este es el caso de la Comunidad de Madrid (+ 8\%), la Comunidad Valenciana (+ 88\%), Andalucía (+ 1\%) y el País Vasco (+ 74\%). En Navarra, el aumento del gasto en 2012 se produjo en hogares con ingresos entre 1.000 y $2.000 €$. Cataluña experimentó un ligero descenso (-5\%) en comparación con la disminución de los hogares de mayores ingresos. En cuanto a los gastos en servicios de transporte público, el 
gasto de los hogares también varía con el nivel de ingresos: mientras que los hogares más pobres aumentan el gasto en 2012, en los hogares de mayores ingresos ocurre lo contrario.

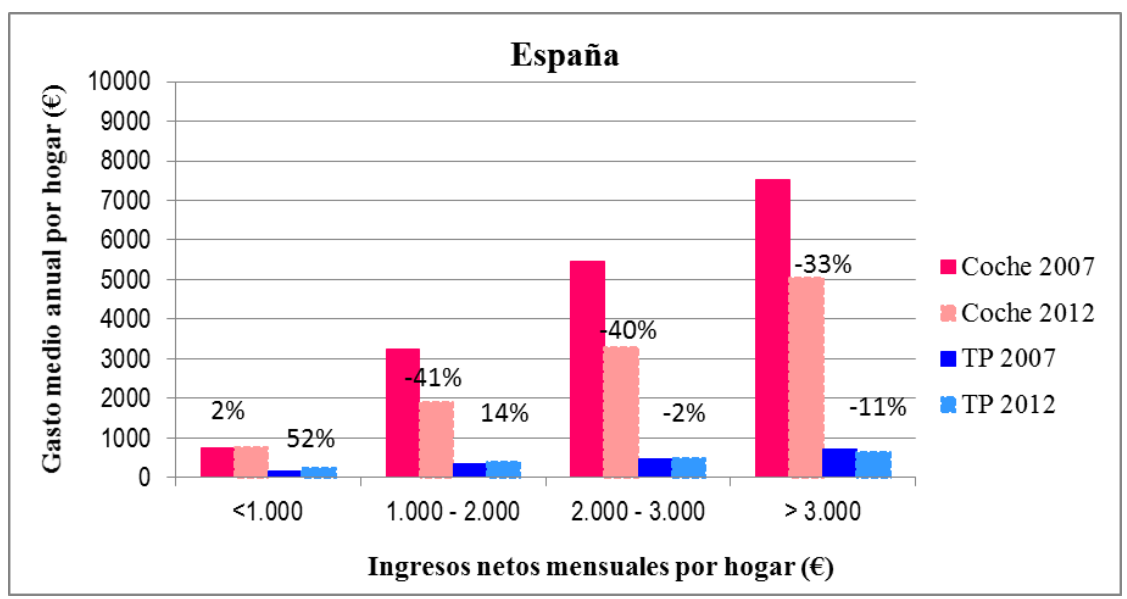

Fig. 3 - Gasto medio anual por hogar en transporte personal y en servicios de transporte público a nivel nacional

\subsection{Análisis individualizado de cada uno de los gastos en transporte}

\section{- Gasto en transporte personal}

El gasto en transporte personal está compuesto por el gasto en compra y uso del transporte personal, es decir, del coche. Se ha visto en la Figura 2 una clara disminución del gasto de los hogares en el transporte personal entre 2007 y 2012 en todas las regiones. El análisis de sus dos componentes por separado pone de manifiesto que esta reducción se debe a la disminución del gasto en la compra de coches. Este resultado está en línea con el hecho de que a medida que aumentan los ingresos disponibles, la gente trata de comprar coches nuevos (Goodwin et al, 2004), o en otras palabras, la reducción de los ingresos del hogar conduce a una reducción en la compra de vehículos. De acuerdo con este resultado, los españoles son propensos a posponer la compra de un coche nuevo y ampliar la vida útil del que tienen en períodos de recesión económica. Esto es cierto en casi todos los hogares españoles, excepto en los que tienen ingresos mensuales menores a $1.000 €$ en el País Vasco.

La Figura 5 muestra el gasto de los hogares en la compra de vehículos en las diferentes regiones y diferentes niveles de ingresos en los años 2007 y 2012. Se puede observar que la desviación respecto a la media nacional es mucho menor en 2012 que en 2007, a excepción de los hogares de mayores ingresos. La Figura 6 muestra el gasto de los hogares en la utilización del transporte personal en las diferentes regiones y diferentes niveles de ingresos, en los años 2007 y 2012. Los hogares andaluces se destacan por su alto gasto en 2007 (874$4.051 €$ por hogar), superior a la media nacional (523-4.019 € por hogar) y a las demás regiones para todos los niveles de ingresos, excepto el más alto. Por el contrario, Cataluña, el País Vasco y Navarra no alcanzan la media nacional en ninguno de los niveles de ingresos. En 2012, los hogares andaluces siguieron siendo notables por sus elevados gastos, pero no tan marcadamente como en 2007. Los hogares en el País Vasco y Cataluña siguieron gastando menos que el promedio nacional para todos los grupos de ingresos en ambos años, con gastos que van desde 400 a $3.200 €$ o desde 400 a $3.600 €$ por hogar, respectivamente, dependiendo del nivel de ingreso mensual de los hogares. En cambio, en Navarra, los gastos en utilización del transporte personal aumentaron en 2012 para todos los niveles de ingresos. A pesar de que Navarra y el País Vasco tengan gastos similares en 2007, Navarra registró un aumento mucho mayor en 2012. En la Comunidad de Madrid los gastos en utilización del 
transporte personal estaban muy cerca de la media nacional para ambos años.

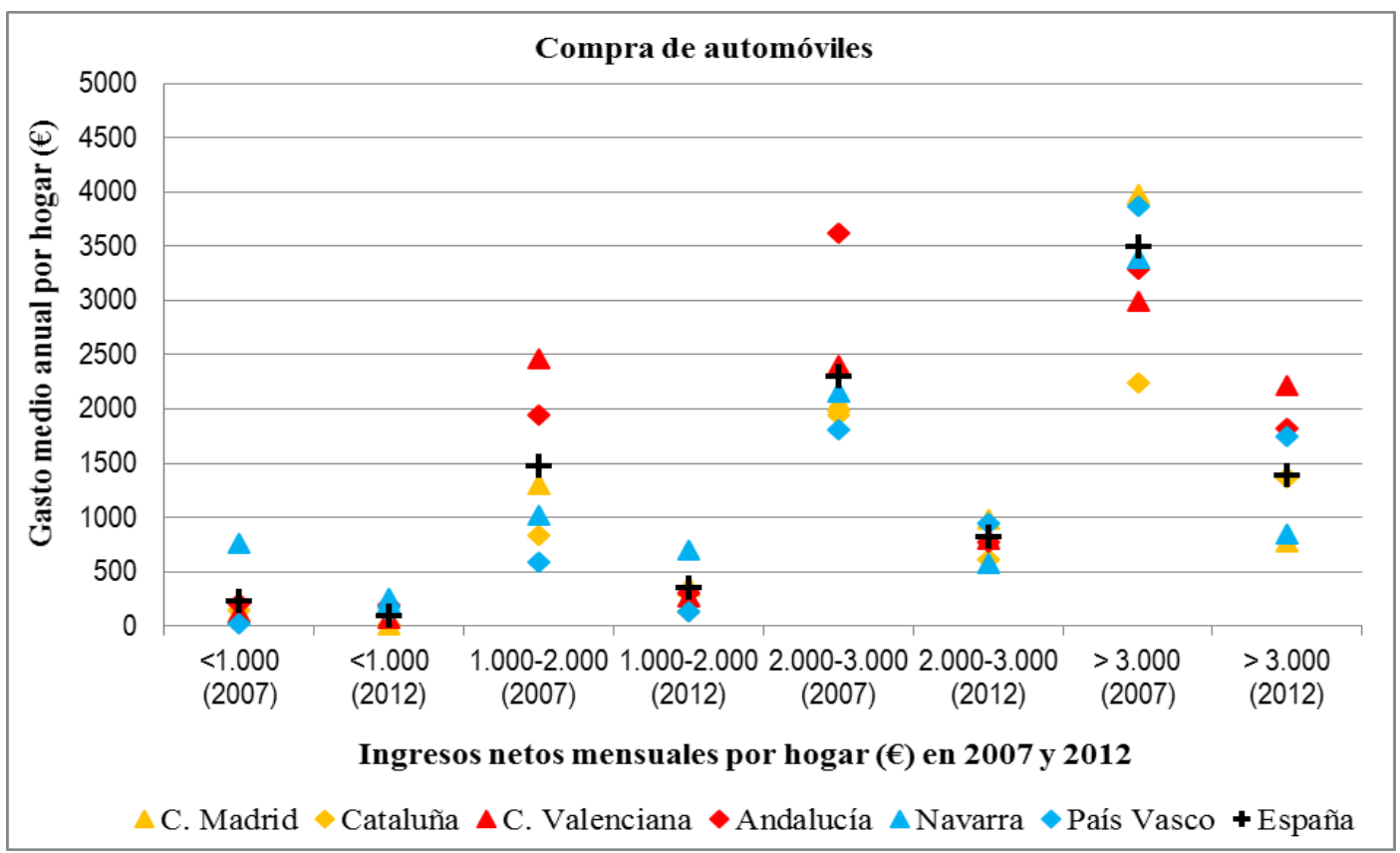

Fig. 4 - Gasto promedio anual por hogar en la compra de vehículos

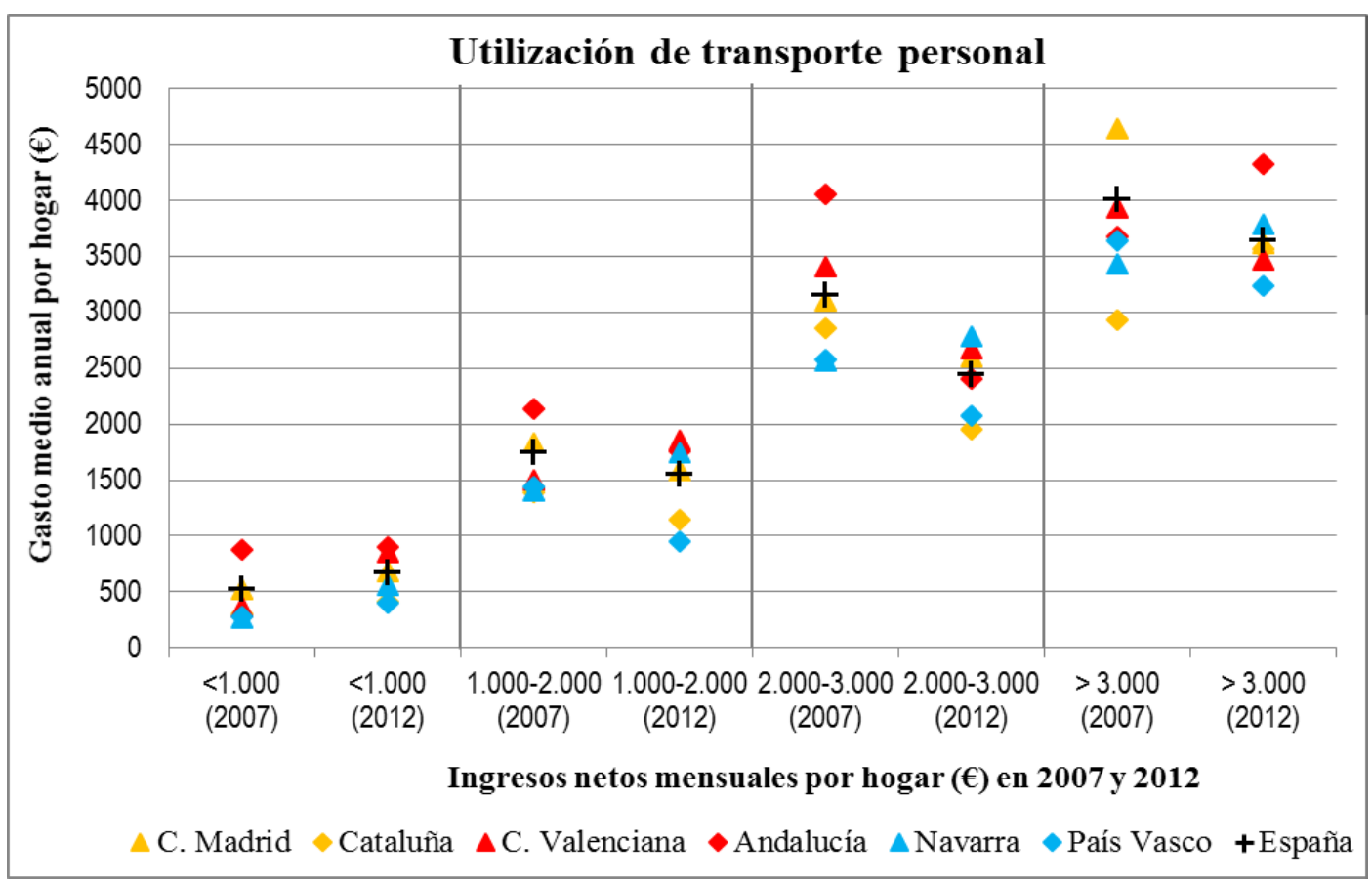

Fig. 5 - Gasto promedio anual por hogar en la utilización de vehículos

\section{- El gasto en servicios de transporte público}

Los resultados de esta sección se centran en el gasto anual de los hogares en los servicios de transporte público urbano y metropolitano en las diversas regiones en 2007 y 2012 (Figura 6). En general, estos gastos son mayores en 2012 en comparación con los de 2007. Martínez et al. (2014) también destacan un aumento en el gasto de las familias españolas en el transporte público terrestre entre 2007 y 2012, a pesar de que no se centran exclusivamente en los municipios de más de 100.000 habitantes. A pesar de este aumento, el gasto de los 
hogares en los servicios de transporte público urbano y metropolitano varía según la región y el nivel de ingresos. Por ejemplo, en todas las regiones se observa que los hogares de menores ingresos aumentaron su gasto en servicios de transporte público entre 2007 y 2012 , entre un $2 \%$ en la Comunidad Valenciana y un $138 \%$ en Cataluña. Además, Andalucía, Navarra, País Vasco y la Comunidad Valenciana muestran valores por debajo de la media nacional en 2007 (100 - $500 €$ por hogar), mientras que la Comunidad de Madrid y Cataluña tienden a estar por encima de ella. A diferencia de otras regiones, los hogares de la Comunidad de Madrid aumentaron su gasto en transporte público urbano y metropolitano, independientemente de su nivel de ingresos.

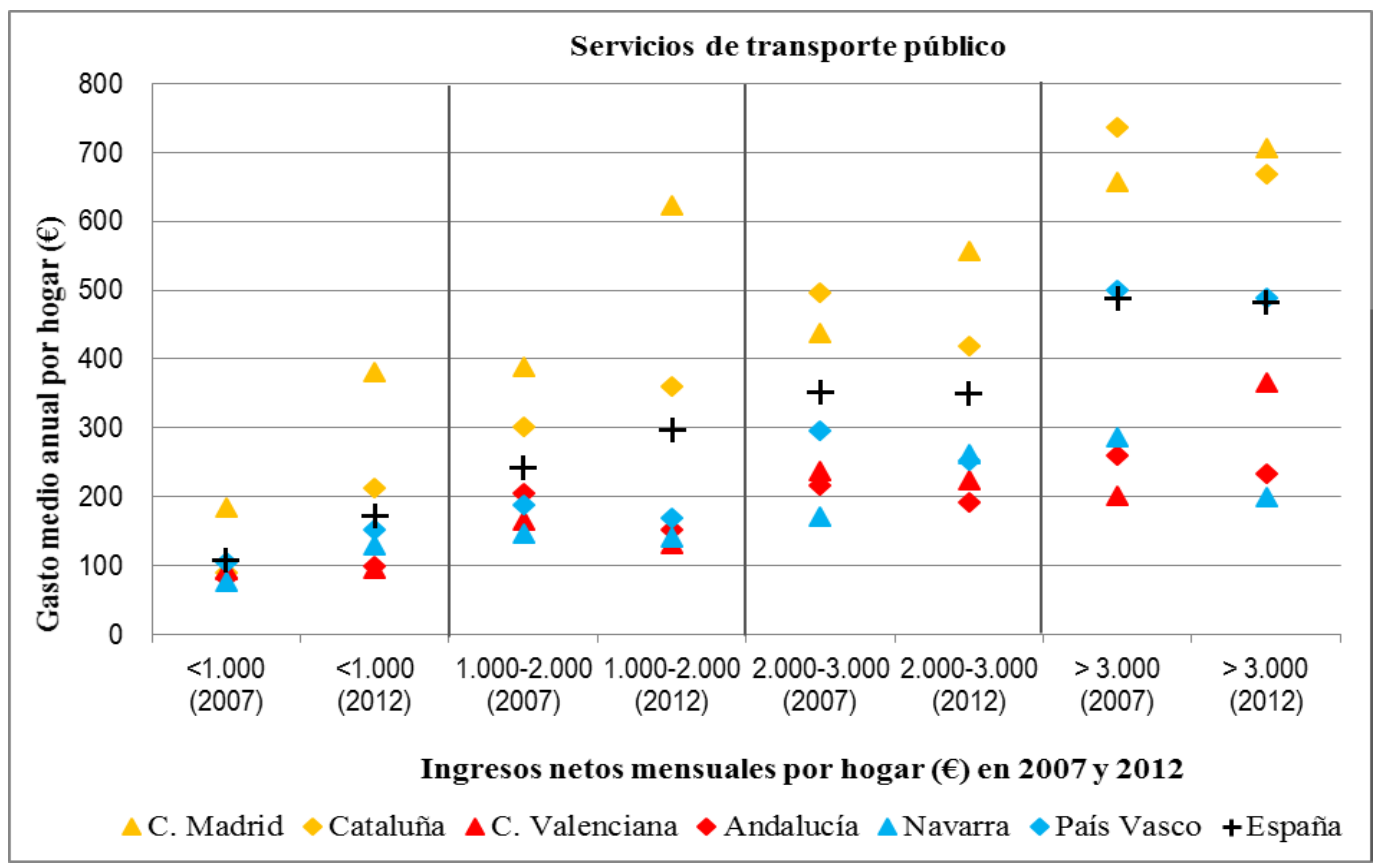

Fig. 6 - Gasto medio anual por hogar en los servicios de transporte público urbano y metropolitano

\section{CONCLUSIONES}

El objetivo de este artículo es analizar cómo la crisis económica ha afectado al gasto en transporte de los hogares españoles entre 2007 y 2012, y ver si existen diferencias en el gasto por regiones o según sus niveles de ingresos. El análisis se realiza sobre la base de los datos procedentes de la encuesta de presupuestos familiares, y se centra en seis regiones españolas.

Una primera conclusión de este análisis es que la proporción del gasto dedicada al transporte personal depende del nivel de ingresos de los hogares, siendo mayor en los hogares con mayores ingresos. Sin embargo, no es cierto para los gastos en servicios de transporte público, ya que la proporción de gasto es independiente del nivel de ingresos del hogar. Así, en los hogares españoles el porcentaje dedicado al transporte se redujo en 2012, en comparación con los de 2007, excepto los hogares con ingresos mensuales netos por debajo de $1.000 €$. Esta disminución se debe principalmente a la reducción de los gastos en el transporte personal, y más concretamente, de los gastos en la compra de vehículos. Por el contrario, los hogares con menos recursos aumentaron su gasto en la utilización del transporte personal en 2012, de media un 29\%. En cuanto a los gastos en servicios de transporte público, en todas las regiones estudiadas los hogares más pobres aumentaron su gasto en 2012 -desde el 2\% en Navarra, al $123 \%$ en la C. Madrid- con un aumento promedio nacional del $52 \%$. 
En conclusión, el gasto de los hogares españoles en transporte depende en gran medida del nivel de ingresos de las familias. La influencia del nivel de ingresos es mayor para los gastos de transporte personal que para los gastos en los servicios de transporte público. Los hogares con menores ingresos son los más afectados en tiempos de crisis, ya que son los que menos pueden de ajustar su gasto, en particular, sus gastos de transporte. Por lo tanto, en periodos de recesión económica son necesarias las políticas sociales, como la implantación de tarifas especiales para los que tienen menos recursos, para ayudar a este grupo a mantener su movilidad y evitar las desigualdades y la pérdida de oportunidades que se derivan de una reducción de su movilidad.

\section{REFERENCIAS}

Banco Mundial (2014). PIB per cápita, en http://datos.bancomundial.org/indicador/NY.GDP.PCAP.CD (acceso 26 Febrero 2015).

Bresson, G., Dargay, J., Madre, J.-L. and Pirotte, A. (2003). The main determinants of the demand for public transport: a comparative analysis of England and France using shrinkage estimators, Transp Research Part A - Policy and Practice, 37 (7), 605-627.

Cascajo R., Diaz-Olvera L., Monfort V., Monzón A., Plat D., Ray J-B (2014). Situation de crise et mobilite quotidienne. Le cas de l'Espagne, paper presented at 51ème Colloque d'ASRDLF, 7-9 July, Marne-la-Vallée (France).

Dargay, J. (2001). The effect of income on car ownership: evidence of asymmetry, Transp Research Part A - Policy and Practice, 35 (9), 807-821.

Dargay, J. (2007). The effect of prices and income on car travel in the UK, Transp Research Part A - Policy and Practice, 41 (10), 949-960.

EC (2009). Economic Crisis in Europe: Causes, Consequences and Responses, available at: http://ec.europa.eu/economy_finance/publications/publication15887_en.pdf.

$\begin{array}{lll}\text { Eurostat (2014). } & \text { Statistics } & \text { Explained, }\end{array}$ http://epp.eurostat.ec.europa.eu/statistics_explained/index.php/Main_Page (acceso 2 Febrero 2016)

Frondel, M. and Vance, C. (2011). Rarely enjoyed? A count data analysis of ridership in Germany's public transport, Transport Policy, 18 (2), 425-433.

Goodwin, P., Dargay, J., \& Hanly, M. (2004). Elasticities of road traffic and fuel consumption with respect to price and income: a review, Transport Reviews, 24(3), 275292.

Holmgren, J. (2007). Meta-analysis of public transport demand, Transportation Research Part A - Policy and Practice, 41 (10), 1021-1035.

INE-Instituto Nacional de Estadística, http://www.ine.es/inebmenu/indice.htm

Martínez, Ó., Barea, P. and García, J. M. (2014). El gasto de las familias españolas en transporte, Asintra (144), 46-51.

Monzón, A., Cascajo, R., Alonso, A., García, A. and Tadeo, A. (2014). Informe del Observatorio de la Movilidad Metropolitana 2012, Ministerio de Agricultura, Alimentación y Medio Ambiente.

Nijkamp, P. and Pepping, G. (1998). Meta-analysis for explaining the variance in public transport demand elasticities in Europe, Journal of Transportation and Statistics, 1 (1), $1-14$.

Paulley, N., Balcombe, R., Mackett, R., Titheridge, H., Preston, J., Wardman, M., Shires, J. and White, P. (2006). The demand for public transport: The effects of fares, quality of service, income and car ownership, Transport Policy, 13 (4), 295-306.

Taylor, B. D., Miller, D., Iseki, H. and Fink, C. (2009). Nature and/or nurture? Analyzing the determinants of transit ridership across US urbanized areas, Transportation Research Part A-Policy and Practice, 43 (1), 60-77. 